\title{
Chemical Constituents and Pharmacological Properties of Poria cocos
}

Author

Affiliation
José-Luis Ríos

Department of Pharmacology, University of Valencia, Valencia, Spain
Key words

- Poria cocos

- Polyporaceae

- polysaccharides

- triterpenes

- anti-inflammatory activity

- anticancer activity

- immunomodulatory activity received Nov. 19, 2010

revised January 25, 2011

accepted January 31, 2011

\section{Bibliography}

Dol http://dx.doi.org/

10.1055/s-0030-1270823

Published online February 23,

2011

Planta Med 2011; 77: 681-691

(c) Georg Thieme Verlag KG

Stuttgart · New York .

ISSN 0032-0943

\section{Correspondence}

Prof. Dr. José-Luis Ríos

Department of Pharmacology

University of Valencia

Av. Vicent Andres

Estelles $\mathrm{s} / \mathrm{n}$

46100 Burjassot

Valencia

Spain

Phone: + 34963544973

Fax: + 34963544998

riosjl@uv.es

\section{Abstract}

$\nabla$

Poria cocos (Polyporaceae) is a saprophytic fungus that grows in diverse species of Pinus. Its sclerotium, called fu-ling or hoelen, is used in traditional Chinese and Japanese medicine for its diuretic, sedative, and tonic effects. Various studies of this fungus have demonstrated its marked anti-inflammatory activity in different experimental models of acute and chronic inflammation. It is widely used as a constituent of many preparations in Asian medicine, but the number of research papers on its clinical properties is insufficient for establishing its efficacy and safety from a scientific point of view. In this review, we have compiled all the published data concerning the chemistry, pharmacology, and clinical uses of this drug in order to evaluate its clinical interest for future use against various pathologies in which inflammation and immunodepression are implicated. We selected the papers for review on the basis of their ethnopharmacological relevance, using the most relevant databases for the biomedical sciences. Studies on various fungus extracts as well as on the major phytochemical compounds (polysaccharides and triterpenoids) present in Poria cocos comprised the principal objectives of this review. In several of the studies reviewed, the inhibitory effects of triterpenes on phospholipase $A_{2}\left(P_{2} A_{2}\right)$ have been clearly demonstrated. In addition, the inhibitory effects of Poria cocos on the secretion of different cytokines from human peripheral blood monocytes have also been described. Triterpenoids are known to have a pivotal influence on certain diseases such as rheumatoid arthritis, psoriasis, autoimmune uveitis, septic shock, and possibly bronchial asthma, while polysaccharides can potentiate the immune response. Reviewing the literature, we found that polysaccharides from Poria cocos enhanced the secretion of immune stimulators and suppressed the secretion of immune suppressors, thus potentiating the immune response.
In addition, they showed antitumor activity against different cancer cell lines. This activity is associated with their capacity to inhibit angiogenesis by downregulating both NF- $k$ B and the induction of NF- $k \mathrm{~B} /$ Rel translocation.

\section{Abbreviations \\ $\nabla$}

AAPH: $\quad 2,2$ '-azobis(2-amidinopropane) dihydrochloride

Akt: a serine/threonine specific protein kinase family

DMBA: 7,12-dimethylbenz[a]anthracen

EBV-EA: Epstein-Barr virus early antigen

Erk: extracellular signal-regulated kinase

GBM: glomerular basement membrane

GM-CSF: granulocyte-monocyte colony stimulating factor

H-ras: human gene that encodes proteins involved in the regulation of cell division in response to growth factor stimulation

HBV: hepatitis B virus

$\mathrm{ID}_{50}$ : inhibitory dose-50

IFN: interferon

IL: interleukin

PARP: poly(ADP-ribose)-polymerase

PCM: $\quad$ Poria cocos mycelia

PCP: $\quad$ Poria cocos protein

PCPS and

PC-PS: Poria cocos polysaccharide

PCSC: polysaccharide from Poria cocos sclerotium

$\mathrm{PLA}_{2}$ : $\quad$ phospholipase A

PPAR: peroxisome proliferator-activated receptor

TGF: transforming growth factor

TNF: tumor necrosis factor

TPA: 12-O-tetradecanoylphorbol 13-acetate

TLR4: Toll-like receptor 4 


\section{Taxonomical Classification and Biological Relevance} $\nabla$

Poria cocos (Schw.) Wolf (Polyporaceae) is known by different botanical synonyms, such as Wolfiporia extensa (Peck) Ginns, Wolfiporia cocos (F.A. Wolf) Ryvarden \& Gilb., Daedalea extensa Peck, Macrohyporia extensa (Peck) Ginns \& J. Lowe, Macrohyporia cocos (Schwein.) I. Johans. \& Ryvarden, Sclerotium cocos Schwein., and Pachyma cocos Fr. The genus is made up of species that possess simple septate hyphae, cause brown rots, and produce annual polyporoid fruiting bodies with hyaline spores [1]. These shared morphological and physiological characteristics have long been considered important in traditional polypore taxonomy; however, recent molecular work indicates that this genus falls within the "core polyporoid" class of true polypores [2]. The species of this genus show microscopic similarities to other related species from closely related genera, such as Laetiporus, Phaeolus, and Pycnoporellus, despite producing macroscopic fruiting bodies that are thin, resupinate, and lacking in bright pigmentations [1].

Poria cocos is dimitic and has greatly inflated skeletal hyphae, a defining characteristic of this genus [3]. This species gives large, edible sclerotia that have been referred to as "tuckahoes" or "Indian bread" in North America, where the name Wolfiporia cocos is used. However, the name Poria cocos is preferred in Asia [1], where the sclerotium - called fu-ling in Chinese and hoelen in Japanese - is collected and used in traditional Chinese and Japanese medicine as a diuretic, sedative, and tonic [4].

Poria cocos is a saprophytic fungus that grows in diverse Pinus species such as $P$. densiflora and $P$. mansoniana. In the wild it grows much like the European truffle, but other genera, such as Citrus, Eucalyptus, or Quercus, can be parasited [5]. Different parts of fu-ling are used in herbal medicine: the bark or fu-ling$p i$, the outermost layer next to the bark or chih-fu-ling, which is reddish in color, the middle layer or bai-fu-ling (or simply fuling), which is white in color, and the core or fu-shen, which includes the wood to which it is attached. Each crude drug obtained from Poria cocos has different properties [6].

\section{Chemical Composition}

$\nabla$

Poria cocos contains two principal groups of chemicals, the triterpene fraction and the polysaccharide fraction. Other minor compounds have also been described, including steroids, amino acids, choline, histidine, and potassium salts [5-7].

\section{Triterpenes}

Many triterpenes have been isolated from Poria cocos, almost all of them derived from lanostane or secolanostane skeletons. During the past decade, Tai et al. [8-13] and other Chinese and Japanese research groups have isolated all the major known compounds from Poria cocos, including various triterpenes ( Table 1). Recently, Akihisa et al. [14,15] isolated 35 compounds, 20 previously known and 15 new structures from this species, while Zheng and Yang $[16,17]$ isolated 10 triterpenes, two of which poriacosone $A$ and poriacosone $B$ - were new.

The isolated triterpenes from Poria cocos could be considered derivatives from a lanostane skeleton; however, some differences have been observed. For example, many possess different structures derived from an eburicane skeleton ( $\mathrm{a}_{21}$ lanostane with a methyl in $C_{24}$ ) and they usually present one (at $C_{8}-C_{9}$ ) or two (at $\mathrm{C}_{7}-\mathrm{C}_{8}$ and $\mathrm{C}_{9}-\mathrm{C}_{11}$ ) unsaturated positions. Moreover, various compounds are derived from a 3,4-seco-lanostane and 3,4-seco-ebur-
Table 1 Triterpenes isolated from Poria cocos.

\begin{tabular}{|c|c|}
\hline Lanostane-type triterpenes & References \\
\hline Trametenolic acid & {$[10]$} \\
\hline Dehydrotrametenolic acid (4) & [18] \\
\hline 3-epi-Dehydrotrametenolic acid & {$[14]$} \\
\hline $16 \alpha$-Hydroxytrametenolic acid & [19] \\
\hline 3-O-Acetyl-16 $\alpha$-hydroxytrametenolic acid (5) & [12] \\
\hline 3-0-Acetyl-16 $\alpha$-hydroxydehydrotrametenolic acid & [12] \\
\hline 16 $\alpha$-27-Dihydroxydehydrotrametenoic acid & {$[15]$} \\
\hline Dehydrotrametenonic acid (12) & {$[20]$} \\
\hline $3 \beta, 16 \alpha$-Dihydroxylanosta-7,9(11),24-trien-21-oic acid & [19] \\
\hline \multicolumn{2}{|l|}{ Eburicane-type triterpenes } \\
\hline Eburicoic acid & [21] \\
\hline Dehydroeburicoic acid (17) & {$[10]$} \\
\hline $16 \alpha-25$-Dihydroxydehydroeburicoic acid & [14] \\
\hline Dehydroeburiconic acid (11) & [14] \\
\hline $16 \alpha$-Hydroxyeburiconic acid & {$[14]$} \\
\hline 16 $\alpha$-25-Dihydroxydehydroeburiconic acid & [15] \\
\hline Pachymic acid (1) & [21] \\
\hline Dehydropachymic acid (14) & [9] \\
\hline 3-epi-Dehydropachymic acid & [12] \\
\hline $16 \alpha$-Hydroxydehydropachymic acid & [19] \\
\hline 25-Hydroxypachymic acid & [16] \\
\hline Tumulosic acid (15) & {$[21]$} \\
\hline Dehydrotumulosic acid (2) & {$[21]$} \\
\hline 3-epi-Dehydrotumulosic acid (16) & [11] \\
\hline $15 \alpha$-Hydroxydehydrotumulosic acid & [14] \\
\hline 25-Hydroxy-3-epi-tumulosic acid & [15] \\
\hline 25-Hydroxy-3-epi-hydroxytumulosic acid & [11] \\
\hline 3 $\beta$-Hydroxybenzoyldehydrotumulosic acid & [18] \\
\hline $5 \alpha-8 \alpha$-Peroxydehydrotumulosic acid & [14] \\
\hline Polyporenic acid C (13) & [21] \\
\hline 6a-Hydroxypolyporenic acid C (18) & {$[22]$} \\
\hline 29-Hydroxypolyporenic acid C & {$[16]$} \\
\hline Poriacosone A & [17] \\
\hline Poriacosone B & [17] \\
\hline \multicolumn{2}{|l|}{ seco-Lanostane-type triterpenes } \\
\hline Poricoic acid B (3) & [8] \\
\hline 16-Deoxyporicoic acid B (8) & [14] \\
\hline Poricoic acid E & [11] \\
\hline Poricoic acid BM & [11] \\
\hline Poricoic acid G (6) & [23] \\
\hline Poricoic acid GM & {$[15]$} \\
\hline \multicolumn{2}{|l|}{ seco-Eburicane-type triterpenes } \\
\hline Poricoic acid A (7) & [8] \\
\hline Poricoic acid C (9) & [14] \\
\hline Poricoic acid D & {$[14]$} \\
\hline Poricoic acid $\mathrm{F}$ & [11] \\
\hline Poricoic acid H & {$[23]$} \\
\hline Poricoic acid AM & {$[14]$} \\
\hline Poricoic acid CM & [14] \\
\hline Poricoic acid DM & [14] \\
\hline Poricoic acid HM & [15] \\
\hline 6,7-Dehydroporicoic acid H & [15] \\
\hline 25-Hydroxyporicoic acid C & [15] \\
\hline 25-Hydroxyporicoic acid H & [14] \\
\hline 26-Hydroxyporicoic acid DM & [15] \\
\hline 25-Methoxyporicoic acid A (10) & [15] \\
\hline
\end{tabular}

icane skeleton. These general structures are given in 0 Fig. 1, with the most relevant active triterpenoids being shown in - Fig. 2 and Table 1. 

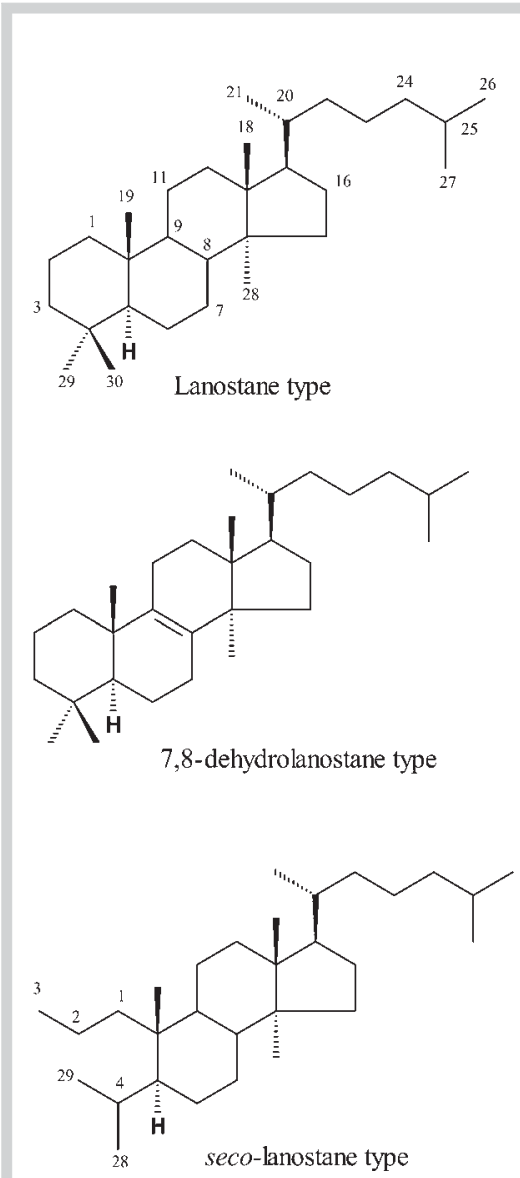

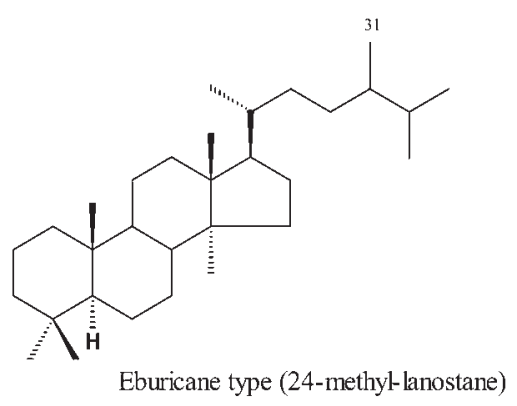

Fig. 1 General structures of triterpenoids from Poria cocos.

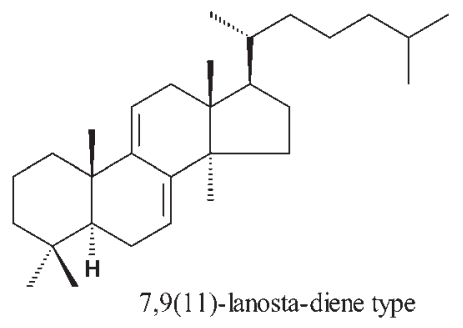

\section{Polysaccharides}

Many different polysaccharides have been isolated from Poria cocos; however, because the nomenclature is not sufficiently clear, there are probably several names for the same compound. Before 1980 , various authors had already isolated a compound called $\beta$ pachyman which was defined as $(1 \rightarrow 3)-(1 \rightarrow 6)-\beta$-D-glucan [24]. Other authors subsequently obtained the derivatives later dubbed pachymaran, carboximethyl pachymaran, U-pachymaran f, and polysaccharide $\mathrm{H}_{11}$, which exhibited different properties when they were tested and thus probably have different chemical configurations [25]. More recently, other authors have isolated different polysaccharides from Poria cocos; because these were more clearly defined, their chemical structure could be established unequivocally. For example, six polysaccharide fractions were isolated sequentially from the mycelia of a wild strain of Poria cocos cultured in two media differing in one constituent, either bran extract or corn steep liquor, labeled wb and wc, respectively. From these, various extracts were obtained with $0.9 \% \mathrm{NaCl}$ (PCM1), hot water (PCM2), $0.5 \mathrm{NaOH}$ (PCM3 I and II), and $88 \%$ formic acid (PCM4 I and II). The heteropolysaccharides wb- and wcPCM1 and PCM2 were mainly composed of $\alpha$-D-glucose, mannose, and galactose, whereas wb-PCM3-I and wc-PCM3-I were mainly ( $1 \rightarrow 3$ )- $\alpha$-D-glucans. For their part, wb- and wc-PCM3-II, PCM4-I, and PCM4-II were all ( $1 \rightarrow 3)-\beta$-D-glucans. In addition, two exo-polysaccharides isolated from the two culture media via methanol precipitation ( $\mathrm{wb}$ - and wc-PCM0) also differed in their monosaccharide composition [26].

Recently, Wang et al. [27] isolated six polysaccharides from the sclerotium of Poria cocos, naming them PCS1, PCS2, PCS3-I,
PCS3-II, PCS4-I, and PCS4-II, depending on their solubility and molecular weights. PCS1, PCS2, and PCS3-I were identified as heteropolysaccharides containing D-glucose, D-mannose, D-fucose, and traces of D-xylose. PCS3-I is a protein-bound heteropolysaccharide while PCS3-II is a linear $(1 \rightarrow 3)-\beta$-D-glucan of high purity that constitutes the main component of the sclerotium of Poria cocos. PCS4-I is a linked branched $(1 \rightarrow 3)-\beta$-D-glucan with few $\beta$ - $(1 \rightarrow 6)$, and PCS4-II is a linked branched $(1 \rightarrow 3)$ $\beta$-D-glucan with few $\beta$ - $(1 \rightarrow 2)$ and $\beta$ - $(1 \rightarrow 6)$. However, in a pilot scale facility-fermentation tank, a water-insoluble derivative with $\alpha$ configuration was isolated from Poria cocos mycelia, $(1 \rightarrow 3)-\alpha$-D-glucan, which was coded as Pi-PCM3-I [28].

\section{Other isolated compounds}

Ukiya et al. [23] described the isolation of dehydroabietic acid methyl ester, Akihisa et al. [15] that of 7-oxo-dyhydroxydehydroabietic acid, and Li et al. [29] the isolation of (S)-(+)-turmerone and ergosterol peroxide, along with other known triterpenoids. Other nonrelevant compounds have also been reported in Poria cocos, including hyperin, ergosterol, choline, histidine, and potassium salts [6,7], along with 15 amino acids [5].

\section{Pharmacological Properties \\ $\nabla$ \\ Anti-inflammatory activity}

A hydroalcoholic extract from Poria cocos was found to inhibit the acute ear edema induced by 12-O-tetradecanoylphorbol 13acetate (TPA) and arachidonic acid, the paw edema induced by 

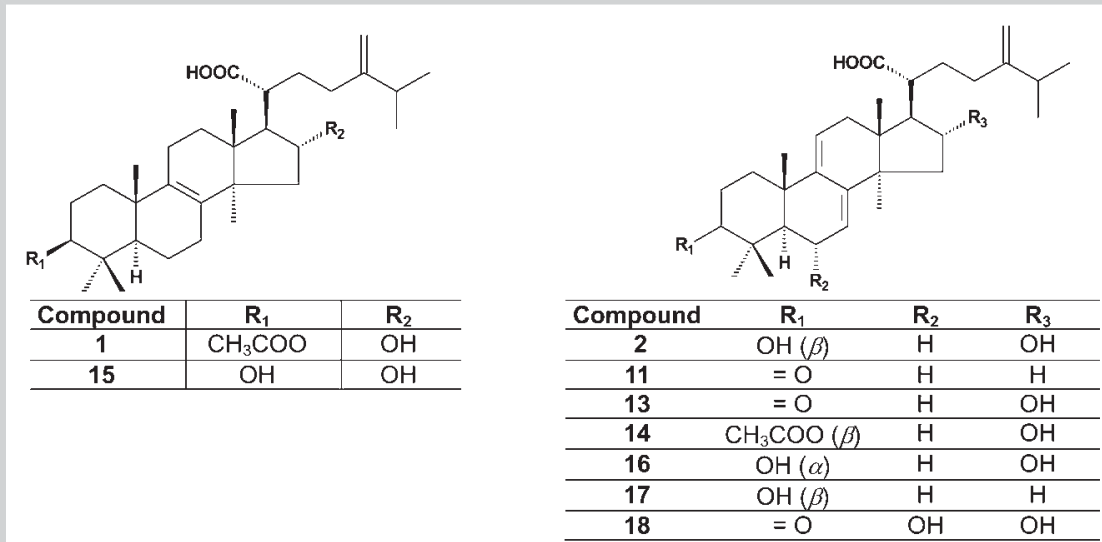

Fig. 2 Active lanostanoids isolated from Poria cocos.
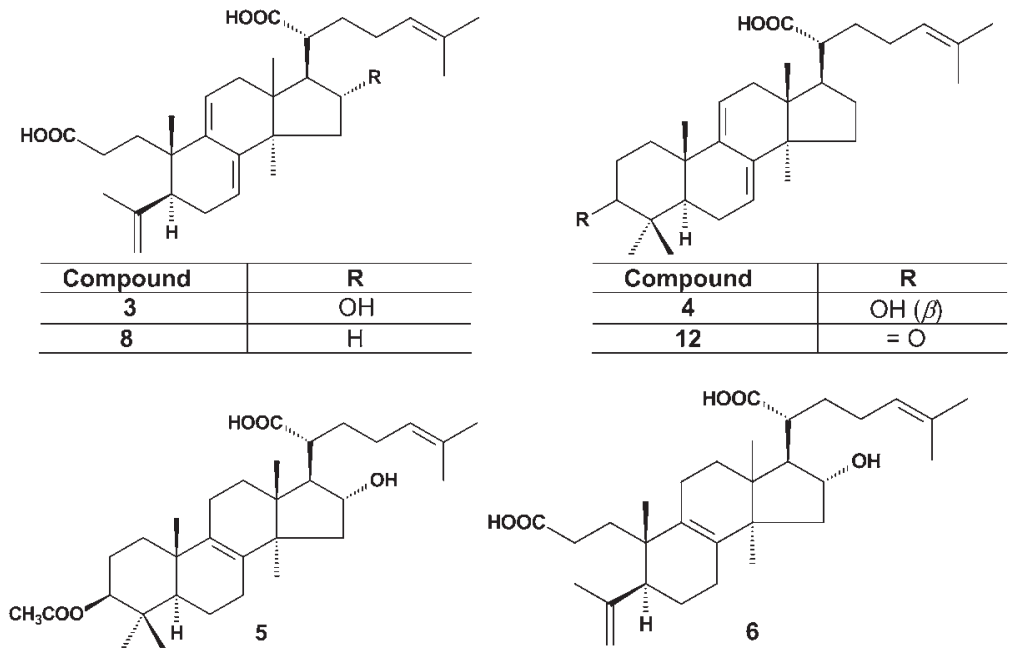

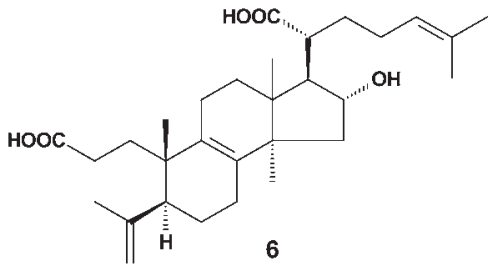<smiles>[R6]C1C[C@H]2[C@@H]3C=C[C@H](C(=C)C)[C@@](C)(CCC(=O)O)C3=CC[C@]2(C)[C@H]1[C@H](CCC(=C)C([R2])(C)C)C(=O)O</smiles>

\begin{tabular}{c|c|c}
\hline Compound & $\mathbf{R}_{\mathbf{1}}$ & $\mathbf{R}_{\mathbf{2}}$ \\
\hline $\mathbf{7}$ & $\mathrm{OH}$ & $\mathrm{H}$ \\
\hline $\mathbf{9}$ & $\mathrm{H}$ & $\mathrm{H}$ \\
\hline $\mathbf{1 0}$ & $\mathrm{OH}$ & $\mathrm{OCH}_{3}$ \\
\hline
\end{tabular}

carrageenan, the dermatitis induced by TPA, and the delayedtype hypersensitivity induced by oxazolone. Bioguided isolation of the active compounds led to the identification of two triterpenoids: pachymic acid (1) and dehydrotumulosic acid (2). These isolated compounds inhibited acute ear edema with $\mathrm{ID}_{50}$ values of 4.7 and $0.68 \mathrm{nmol} /$ ear, respectively, which indicates a considerable anti-inflammatory potency [30].

Both compounds also inhibited the acute paw edema induced by phospholipase $\mathrm{A}_{2}\left(\mathrm{PLA}_{2}\right)$ and serotonin while 2 reduced the ear edema induced by ethyl phenylpropiolate. Neither compound had any affect against arachidonic acid-induced acute ear edema. When compounds $\mathbf{1}$ and $\mathbf{2}$ were studied as possible corticoid-like agents, results showed a clear difference between them. Whereas the anti-edema activity of $\mathbf{2}$ was not affected by progesterone (a corticoid receptor antagonist), actinomycin D (an RNA transcription blocker), or cycloheximide (a protein synthesis inhibitor), the activity of 1 was affected by progesterone and, to a lesser degree, by actinomycin $\mathrm{D}$. These findings suggest that while the compounds do not act through a corticoid-like mechanism, in the case of 1 , the corticoid receptor may be implicated [31]. The same authors reported the possible mechanism of action of the major triterpenoids isolated from the active extract. Compounds 1 and $\mathbf{2}$ both showed significant inhibitory activity against $\mathrm{PLA}_{2}$ from Naja naja venom, with the latter exhibiting greater activity [32]. Extracellular PLA 2 plays a pathogenic role both by causing direct damage to the cellular membrane through phospholipid hydrolysis and through the liberation of arachidonic acid, which is the precursor of many of the eicosanoids responsible for inflammatory responses. As such, $\mathrm{PLA}_{2}$ may play a pivotal role in various diseases such as rheumatoid arthritis or psoriasis, thus making these triterpenoids potentially interesting as therapeutic agents. In similar work, Jain et al. [33] demonstrated that acidic 
triterpenoids are inhibitors of PLA $A_{2}$. They studied the effects of two derivatives, masticadienoic and masticadienolic acids, with regard to their interaction with three different forms of $\mathrm{PLA}_{2}$ and demonstrated that both a bulk tetracyclic structure and a side alkyl carboxylic chain are necessary for rendering PLA $\mathrm{A}_{2}$ inactive, although the latter part of the molecule seems to be the one that occupies and blocks the catalytic site of the enzyme. This hypothesis was supported by theoretical spatial considerations as well as by the fact that their homologous methyl esters are inactive. In the case of pachymic and dehydrotumulosic acids, the position of the free carboxylic group can vary $\left(C_{21}\right.$ instead of $\left.C_{26}\right)$ and the hybridization state of carbons $\mathrm{C}_{7}-\mathrm{C}_{10}$ can also admit some variation with no loss of activity. Cuéllar et al. [32] thus hypothesized that the alicyclic structure probably does not exert any spatially-specific function, but merely provides the lipophilicity needed to go along the hydrophobic channel leading to the active site of PLA 2 , as postulated by Scott et al. [34].

In parallel, a second research group studied the effects of various isolated triterpenes on TPA-induced ear edema, obtaining similar results. For example, all the tested compounds inhibited the ear edemas induced by TPA and arachidonic acid, with similar potency values. They also tested a larger group of compounds, including seco-derivatives. Of these, poricoic acid B (3), showed the highest potency in the TPA test while dehydrotrametenolic acid (4) gave the best results in the arachidonic acid test $[18,35]$. In a previous study, Nukaya et al. [19] obtained similar results for the same or related compounds isolated from Poria cocos.

Prieto et al. [36] reported the inhibition of leukotriene $B_{4}$ release by 1 and 2 that had previously been isolated from Poria cocos, with this effect being due to the inhibition of PLA 2 rather than to a 5-lipoxygenase effect, as was reported by Cuéllar et al. [32].

Fuchs et al. [37] studied the effects of three concentrations of Poria cocos in a base cream which was tested on experimentally induced irritant contact dermatitis in a repeated sodium lauryl sulphate irritation model. Anti-inflammatory activity was reported for Poria cocos in all three experimental methods when the extracts were applied parallel to the induction period of irritant contact dermatitis. This effect can be explained by the influence of containing product on proinflammatory enzymes, such as $\mathrm{PLA}_{2}$, as previously reported by Cuéllar et al. [32]. These authors had demonstrated the effects of an ethanol extract of Poria cocos on an experimental model of contact dermatitis induced by oxazolone. Since both $\mathbf{1}$ and $\mathbf{2}$ were isolated from this extract, the activity was associated with them [30].

\section{Immunomodulatory properties}

Certain medicinal plants and fungi, among them Poria cocos, are what are commonly referred to as immunomodulators, in that they alter the activity of immune function through the dynamic regulation of informational molecules such as cytokines [38]. This property explains the effects of these plants on the immune system and other tissues. The most relevant research in this field is perhaps that of Yu and Tseng [39], who demonstrated, for example, that a $50 \%$ hot ethanol extract from Poria cocos increased the secretion of interleukin (IL)- $1 \beta$ and IL- 6 in human peripheral blood monocytes in vitro in a dose-dependent manner $6 \mathrm{~h}$ after treatment. At a concentration of $0.4 \mathrm{mg} / \mathrm{mL}$, the extract caused an increase in other cytokines, including tumor necrosis factor (TNF)- $\alpha$. However, at $0.2 \mathrm{mg} / \mathrm{mL}$ it was found to suppress the secretion of transforming growth factor (TGF)- $\beta 3 \mathrm{~h}$ after in vitro treatment. Four years before, Tseng and Chang [40] had demonstrated that a culture medium containing $10 \%$ of a Poria cocos ex- tract obtained with absolute ethanol inhibited IL- $1 \beta$, IL-6, TNF- $\alpha$, and granulocyte-monocyte colony stimulating factor (GM-CSF) secretion from the monocyte monolayer; in contrast, a reduction in the Poria cocos content of the extract produced an increase in cytokine secretion.

The increased secretion of IL- $1 \beta$, IL- 6 , and TNF- $\alpha$ by activated macrophages potentiates the immune response. The inflammatory response is also associated with high levels of these three cytokines in serum secreted by activated neutrophils, because these three cytokines, which are secreted by activated neutrophils and which stimulate the mononuclear phagocytes, are involved in fever and the acute phase of inflammation. TGF- $\beta$, on the other hand, suppresses the inflammatory response by inhibiting macrophage activation and the secretion of other cytokines [39]. Because the extract obtained from Poria cocos enhances the secretion of immune stimulators (IL- $1 \beta$, IL- 6 , and TNF- $\alpha$ ) and suppresses the secretion of an immune suppressor (TGF- $\beta$ ), it should serve to potentiate the immune response [39].

From the dried sclerotium of Poria cocos, Chang et al. [41] isolated and purified a new immunomodulatory protein (PCP), which has a total of $35.6 \mathrm{kDa}$ and consists of a disulfide-linked heterodimeric glycoprotein with two subunits with 14.3 and $21.3 \mathrm{kDa}$, respectively, and which exhibits $\mathrm{N}$ - and $\mathrm{O}$-glycosylation. In vitro, this glycoprotein stimulated RAW 264.7 macrophages through the induction of TNF- $\alpha$ and IL- $1 \beta$ as well as through the regulation of NF- $k$ B-related gene expression. In primary mouse macrophages, PCP directly activated peritoneal cavity macrophages to induce Toll-like receptor 4 (TLR4)-mediated myeloid differentiation factor 88 (MyD88)-dependent signaling. Moreover, the glycosylated portion of PCP was a key factor in PCP signaling through TLR4 in peritoneal macrophages. This glycoprotein, along with Poria cocos itself, could thus be considered a new, potential immunostimulant.

From the dried sclerotia powder of Poria cocos, Chen et al. [42] obtained a $\beta$ - $(1 \rightarrow 3)$-D-glucan coded as PCS3-II, from which they then obtained the corresponding carboxymethylated derivative (C-PC3-II) and the carboxymethylated-sulfated derivative (CS-PCS3-II). The immunopotentiation activity of all three compounds was evaluated and the activity-structure relationship was determined. CS-PCS3-II was found to enhance the immune ability of the spleen and to increase the carbon clearance index of macrophages, the spleen and thymus weight index, hemolytic activity, spleen antibody production, and the delayed type hypersensitivity response in mice. The authors hypothesized that the coexistence of carboxymethyl and sulfate groups in CS-PCS3-II molecules plays an important role in enhancing the immunomodulatory activity of this particular polysaccharide.

\section{Anticancer properties}

Kaminaga et al. [43] studied the inhibitory effects of lanostanetype triterpene acids from Poria cocos on tumor promotion by TPA in two-stage carcinogenesis in mouse skin, demonstrating that 1, 3, and 3-0-acetyl-16 $\alpha$-hydroxytrametenolic acid (5) all inhibited the promoting effects of TPA on skin tumors after initiation with 7,12-dimethylbenz[a]anthracene (DMBA). In 2002, Ukiya et al. [23] tested the inhibitory effects of 10 triterpenes isolated from Poria cocos on the Epstein-Barr virus early antigen (EBV-EA) activation induced by the tumor promoter TPA in Raji cells. The compounds all displayed activity, but subsequent evaluation of the cytotoxicity of the compounds poricoic acid $G(6)$ and poricoic acid $A(7)$ against human cancer cell lines revealed that the former was significantly cytotoxic to leukemia HL-60 
cells at nM range (although it showed only moderate cytotoxicity against the other cell lines) while the latter compound exhibited only moderate cytotoxicity against all the cancer cell lines tested. Several years later, Akihisa et al. [14] used the same protocol to study a series of triterpenoids in vitro to evaluate their antitumor-promoting activity. All the tested compounds exhibited low cytotoxicity against Raji cells, with 13 showing high inhibitory effects and $\mathrm{IC}_{50}$ values of $195-340 \mathrm{~mol}$ ratio/32 pmol TPA. In the same study, the authors evaluated the inhibitory properties in vivo of two compounds, 16-deoxyporicoic acid B (8) and poricoic acid C (9), in a two-stage carcinogenesis test on mouse skin using DMBA as an initiator and TPA as a promoter. Eleven weeks after treatment, the percentage of papilloma-bearing mice in the treated groups was only $20 \%$ for $\mathbf{8}$ and $27 \%$ for $\mathbf{9}$, whereas after 20 weeks the percentages were $80 \%$ and $87 \%$, respectively. When the authors evaluated the average number of papillomas per mouse at 11 weeks it was 1.2 and 1.5 , respectively, whereas at 20 weeks it was 3.2 and 3.6, respectively. These findings, however, should be compared to those of the untreated group, which showed a $100 \%$ incidence of papillomas 11 weeks after promotion. From the results of both the in vitro EBV-EA induction assay and the in vivo two-stage carcinogenesis test, the authors concluded that the lanostane-type triterpenes from the sclerotium of Poria cocos, especially those hydroxylated at $\mathrm{C}_{16} R$ and/or those with a 3,4-seco-3-oic acid functionality ( $\bullet$ Fig. 1 ), may serve as valuable chemopreventive agents against chemical carcinogenesis. Two years later, the same authors completed this study with 11 compounds isolated from Poria cocos: 10 triterpenoids and 1 diterpene. All showed inhibitory effects in vitro against TPA-induced EBV-EA activation in Raji cells. However, in vivo only 25methoxyporicoic acid A (10) exhibited inhibitory activity in the two-stage carcinogenesis test. The authors concluded that while lanostane triterpenoids may be potentially chemopreventive, they are not effective antitumor agents [15].

Mizushina et al. [44] studied the effects of 9 triterpene acids (4 eburicane-type, 1 lanostane-type, 1 seco-lanostane, and 3 secoeburicane-type triterpenes) from Poria cocos as potential inhibitors of DNA polymerases and DNA topoisomerases from mammals. Among the tested compounds, only dehydroeburiconic acid (11) (described as dehydroebriconic acid in this paper) was detected as a possible inhibitor of DNA topoisomerase II activity, with a moderate inhibitory effect on the activity of other DNA polymerases. Dehydrotrametenonic acid (12) also showed a moderate inhibitory effect against topoisomerase II and a weak effect against all the polymerases tested. These findings suggest that 11 and 12 should be designated as topoisomerase II-preferential inhibitors, with moderate inhibitory effects against all the mammalian DNA polymerases tested. Both compounds also prevented the growth of human gastric cancers, halting them in the $\mathrm{G}_{1}$ phase of the cell cycle. While both compounds have the same pattern of substitution ( $C_{3}$-keto, one carboxyl at $C_{21}$, and no hydroxyl group), 12 has a lanostane skeleton whereas $\mathbf{1 1}$ has an eburicane one. The hydrophobic triterpene backbone and the hydrophilic carboxyl end of these compounds were able to bind to the hydrophobic sheet and the hydrophilic amino acids in DNA polymerase $\beta$, respectively. Both triterpenes showed an interesting spectrum of enzyme inhibition, and were able to recognize small structural differences among the binding sites on topoisomerases. These triterpenes may thus be useful tools for studying the microstructure of such binding sites and could be helpful in designing new drugs with the aid of computer simulation [44].
From a bioactivity-guided fractionation of the methylene chloride extract from the sclerotium of Poria cocos, Li et al. [29] isolated 6 compounds, three of which exhibited moderate cytotoxicity against a human colon carcinoma cell line; however, four of the compounds -1 , polyporenic acid $C$ (13), dehydropachymic acid (14), and tumulosic acid (15) - exhibited inhibitory activity against DNA topoisomerase II, as well as against DNA topoisomerase I. The activity of some of these compounds was related to the induction of apoptosis. For example, 11 and 12 inhibited calf DNA polymerase $\alpha$ and rat DNA polymerase $\beta$ [20] while 4 selectively inhibited the growth of H-ras transformed cells and induced apoptosis [45]. It also regulated the expression of $\mathrm{H}$-ras as well as that of Akt (a serine/threonine specific protein kinase family) and Erk (extracellular signal-regulated kinase), which are the downstream proteins of $\mathrm{H}$-ras signaling pathways. Another isolated compound from Poria cocos, 1, reduced cell proliferation and induced apoptosis in a dose- and time-dependent manner in androgen-insensitive DU145 prostate cancer cells. Compound 1-treatment decreased Bad phosphorylation, increased Bcl-2 phosphorylation, and activated caspase-9 and caspase-3, all of which, taken together, indicate that 1 initiates apoptosis by causing mitochondria dysfunction. Compound 1 also decreased the expression and activation of proteins within the Akt signaling pathway. These results, along with the compound's previously reported inhibition of $\mathrm{PLA}_{2}$ activity, led these authors to hypothesize a relationship between 1 -influenced apoptosis and reduction of both prostaglandin synthesis and Akt activity, as PLA 2 is elevated in prostatic adenocarcinoma and conversion of arachidonic acid to prostaglandins leads to Akt pro-survival activity [46].

Ling et al. [47] studied the effect of 13 on the growth of A549 nonsmall cell lung cancer cells (NSCLC) and demonstrated that it significantly reduces cell proliferation via the induction of apoptosis, as evidenced by sub- $G_{1}$ analysis, annexin V-FITC staining, and increases in the cleavage of procaspase- $8,-3$ and poly(ADPribose)-polymerase (PARP). However, treatment of cells with 13 was not accompanied by either disruption of mitochondrial membrane potential or an increase in the cleavage of procaspase-9. Further, 13-induced apoptosis was inhibited by caspase8 inhibitors, but not by a caspase- 9 inhibitor. It also suppressed the PI3-kinase/Akt signal pathway and enhanced p53 activation. In conclusion, the results suggest that $\mathbf{1 3}$ induces apoptosis through the death receptor-mediated apoptotic pathway, in which the activation of caspase- 8 leads to the direct cleavage of execution caspases without the involvement of mitochondria.

In 2004, Wu et al. [48] isolated 9 triterpenes from Poria cocos and assayed their cytotoxic and antioxidant properties. None of the compounds showed any promising antioxidant activity, in agreement with data previously reported by Schinella et al. [49], but all of them except 15 showed cytotoxicity at different doses against human lung cancer cell line A549 and human prostate cancer cell line DU145 [50].

Several authors have studied the antitumor activity of various polysaccharides isolated from Poria cocos. For example, Kanayama et al. [25] reported the effects of polysaccharide $\mathrm{H}_{11}-\mathrm{a}$ $(1 \rightarrow 3)-(1 \rightarrow 6)-\beta$-D-glucan in a ratio of $4: 1$ each and with a molecular weight of $5 \times 10^{6}$ - against subcutaneous sarcoma 180 at doses of 4 and $8 \mathrm{mg} / \mathrm{kg} \times 10$, but found that it had no effect on ascites sarcoma 180 . The compound had an inhibition ratio of $96 \%$ with respect to the control group. The anticancer activity of Poria cocos, along with its other effects, has been associated with its capacity to inhibit angiogenesis by downregulating NF- $k B$ [51]. This effect has been studied with the polysaccharide fraction, and es- 
pecially with the compound PCSC (polysaccharide from Poria cocos sclerotium). This polysaccharide induces both the translocation of NF- $k \mathrm{~B} /$ Rel into the nucleus and the binding of DNA to its cognate site in the promoter of the iNOS gene of activated macrophages [52]. In a later study, Lee et al. [53] demonstrated that PCSC induces NF- $k$ B/Rel activation and iNOS expression through the CD14, TLR4, and CR3 membrane receptor and through p38 kinase, which is critical in the signal transduction leading to NF- $k \mathrm{~B} /$ Rel activation in murine macrophages.

Jin et al. [26,54] studied 10 water-soluble heteropolysaccharide fractions obtained from Poria cocos mycelia cultured from one wild and one cultivated strain. Both the in vitro and in vivo antitumor activities of the heteropolysaccharides were evaluated and compared. The authors found that the heteropolysaccharides from the wild strain cultured in a medium containing corn steep liquor exhibited the highest antitumor activities against sarcoma 180 in vivo while the heteropolysaccharides from mycelium cultured in media with bran extract did not significantly inhibit tumor growth. Indeed, polysaccharides isolated from the different strains of Poria cocos mycelia (a series of compounds named PCM) generally seemed to have different in vivo and in vitro antitumor activities, depending on their monosaccharide composition, protein content, molecular mass, and chain conformation.

Zhang et al. [55] studied the reported immune-enhancing and antitumor activities of PCM3-II, a purified water-soluble $\beta$-glucan comprising mainly $(1 \rightarrow 3)$ and $(1 \rightarrow 4)$ linkages obtained from the mycelia of Poria cocos. PCM3-II reduced the proliferation and viability of human breast carcinoma MCF-7 cells in vitro in a dosedependent manner, decreasing the cancer-cell growth by $50 \%$ at $400 \mu \mathrm{g} / \mathrm{mL}$. The authors also studied the time effect of PCM3-II and found that the $\beta$-glucan arrested the cell cycle in the $G_{1}$ phase, an effect associated with downregulation of the expression of cyclins $\mathrm{D}_{1}$ and $\mathrm{E}$. In addition, PCM3-II induced the depletion of the anti-apoptotic $\mathrm{Bcl}-2$ protein, but not the pro-apoptotic Bax protein.

A water-insoluble $(1 \rightarrow 3)$ - $\beta$-D-glucan isolated from fresh sclerotium of Poria cocos was sulfated, carboxymethylated, methylated, hydroxyethylated, and hydroxypropylated to afford five watersoluble derivatives, respectively, to be tested against sarcoma 180 tumor cells and a gastric carcinoma cell strain (MKN-45 and SGC-7901), both in vitro and in vivo. Whereas the native $\beta$-glucan showed no antitumor activity, the sulfated and carboxymethylated derivatives exhibited significant antitumor activities against S-180 and gastric carcinoma tumor cells. The authors, Wang et al., hypothesized that these properties are based on good water solubility, relatively high chain stiffness, and the moderate molecular mass of the derivatives in aqueous solution [56]. This explanation is supported by the results of an experiment conducted by Zhang et al. [57] with the water-soluble heteropolysaccharide ac-PCM0 obtained from Poria cocos, which exhibited in vivo antitumor activity and showed a significantly higher enhancement ratio of body weight than 5-fluorouracil, with no cytotoxic effects.

Two kinds of water-insoluble $(1 \rightarrow 3)$ - $\alpha$-D-glucan samples, abPCM3-I and ac-PCM3-I, isolated from different Poria cocos mycelia were sulfated to obtain two series of water-soluble derivatives, ab-PCM3-I-S1-S5 and ac-PCM3-I-S1-S5, respectively. These were tested against sarcoma 180 tumor cells both in vitro and in vivo and displayed a significantly higher activity than those from the native $\alpha$-D-glucans. Different structural features and the higher water solubility of the transformed products may explain the enhanced activity [58].
The neutral polysaccharide fraction PCPS (Poria cocos polysaccharide, mw about $160 \mathrm{kDa}$ ) was studied for its effects on antiproliferation and differentiation of different human leukemic cells in vitro, with the results showing that it suppressed the cell proliferation of U937 and HL-60 cells. It also induced both U937 cells and HL-60 cells to differentiate into mature monocytes/macrophages, which in turn markedly expressed the surface antigens of CD11b, CD14, and CD68. The differentiated U937 and HL-60 cells exhibited physiological functions such as respiratory burst and phagocytosis. Moreover, the levels of interferon (IFN)- $\gamma$ and TNF- $\alpha$ were higher than those observed in the control group. Antibody neutralization tests demonstrated that the growth-inhibitory and differentiation-inducing activities were mainly due to the elevated levels of IFN- $\gamma$ and TNF- $\alpha$, suggesting that PCPS is a biological response modifier rather than a cytotoxic reagent and could constitute a potential alternative in leukemia therapy [59]. As commented above, Chen et al. [42] obtained a $\beta$-( $1 \rightarrow 3)$-D-glucan (PCS3-II) from Poria cocos, along with its semisynthetic derivatives, carboxymethylated (C-PC3-II) and the carboxymethylated-sulfated (CS-PCS3-II). The authors studied the anticancer properties of these compounds in vivo on male BALB/c mice inoculated with sarcoma 180 (S-180) tumor cells. Microscopic examination of the tumor cells revealed signs of necrosis and apoptosis in cells treated with the carboxymethylated-sulfated derivative and enhancement of immune response in the spleen. The authors suggest that CS-PCS3-II exerts this antitumor activity through immunomodulation.

\section{Antihyperglycemic effects}

Various studies have been published on the use of Poria cocos, either alone or in combination with other crude drugs, to treat diabetes. $\mathrm{Li}$ et al. [60] studied the antihyperglycemic effects of a methanol crude extract of Poria cocos on streptozocin-treated $\mathrm{db} / \mathrm{db}$ mice. The extract reduced blood glucose via increased insulin sensitivity. Three fractions - chloroform, ethyl acetate, and water extracts - were obtained from the crude extract, but only the first exhibited any activity. The authors then isolated three terpenoids (1, 2, and $\mathbf{4})$, which they studied using the same protocol while also examining the effect of modifying/activating the peroxisome proliferator-activated receptor (PPAR)- $\gamma$ pathway on the increase of insulin sensitivity. The authors found that neither the active extract nor the isolated triterpenes activated the PPAR$\gamma$ pathway and hypothesized that the compounds may instead affect 5'-AMP activated protein kinase. Further studies have thus been proposed. However, in a previous study, Sato et al. [61] had demonstrated that $\mathbf{4}$ promotes adipocyte differentiation in vitro and acts as an insulin sensitizer in vivo. Indeed, the compound was found to reduce hyperglycemia in mouse models of noninsulin-dependent diabetes mellitus, acting as an insulin sensitizer, as demonstrated in the glucose tolerance test. It was also shown to induce adipose conversion, activate PPAR- $\gamma$ in vitro, and reduce hyperglycemia in animal models of noninsulin-dependent diabetes mellitus. In addition, Su and Huang [62] demonstrated a positive effect of Poria cocos on the PPAR- $\gamma$ pathway, with its ethyl acetate extract reversing the previous suppression of TNF- $\alpha$ on the PPAR- $\gamma$ of human (LX-2) and rat (HSC-T6) cells while enhancing the PPAR- $\gamma$ activities of hepatic stellate cells. The extract also inhibited NF- $k$ B activity induced by TNF- $\alpha$ and attenuated the TNF- $\alpha$-induced protein and mRNA expression of $\alpha$-smooth muscle actin.

Recently, Huang et al. [63] demonstrated that 2, 13, 14, 15, 16, and especially 1 increase glucose uptake, GLU4 gene expression 
at both mRNA and protein levels, and GLU4 translocation in 3T3L1 adipocytes, but displayed no activity on GLU1 expression.

\section{Other pharmacological effects}

The antinephritic effect of the polysaccharide pachyman isolated from Poria cocos on original-type anti-glomerular basement membrane (anti-GBM) glomerulonephritis in rats was investigated by Hattori et al. [64]. The authors demonstrated its effects against original-type anti-GBM nephritis in rats and concluded that the antinephritic mechanisms of pachyman may partly be due to the inhibitory action of this agent on $\mathrm{C} 3$ deposition in the glomeruli.

Dhan et al. [65] reported the effect of carboxymethyl pachymaram from Poria cocos on the anti-hepatitis B virus (HBV) expression from transfected cells.

A hydroethanolic extract from Poria cocos was tested against the epimastigote of a Trypanosoma cruzi Bra $\mathrm{C} 15 \mathrm{C} 2$ clone in vitro. It showed significant effects against the parasite, inhibiting growth by $30 \%$ [66].

Li et al. [67] were the first to demonstrate the nematicidal activity of Poria cocos, which was able to kill more than $90 \%$ of the saprophytic nematode Panagrellus redivivue, the root-knot nematode Meloidogyne arenaria, and the pine nematode Bursaphelenchus xylophilus. From the active extract, three new compounds were isolated and identified as 2,4,6-triacetylenic octane diacid, 2,4,5,6-tetrahydroxyhexanoic acid, and 3,4-dihydroxy-2-keto-nbutyl-2,4,5,6-tetrahydroxyhexanate. The first compound exhibited the highest activity.

In an in vivo study, Zhang et al. [68] demonstrated the anti-rejection effect of the ethanol extract of Poria cocos at two different doses in rats after cardiac allograft implantation. The survival time of donor hearts in the two experimental groups was significantly prolonged by approximately $130 \%$ and $330 \%$ (in days) with respect to the control group. There was a clear reduction of pathological lesions in the two extract-treated groups, with the percentages of CD3+, CD4+, and CD8+ lymphocytes and the CD4+/CD8+ ratio all being significantly lower.

In vitro studies demonstrated that a methanol extract from Poria cocos protected red blood cells from the hemolysis induced by the hydrophilic radical generator 2,2'-azobis(2-amidinopropane) dihydrochloride (AAPH); from this extract, Sekiya et al. [69] isolated five triterpene carboxylic acids - 1, 4, 5, 13, and 3-epi-dehydrotumulosic acid (16) - which were found to inhibit AAPH-induced lysis of red blood cells.

The effect of Poria cocos on cytosolic free calcium concentration in the brain nerve cells of neonatal rats was investigated by Chen et al. [70]. Working with a water extract, the authors demonstrated that the plant increased cytosolic free calcium in a dose-dependent manner, except at high doses. Moreover, the extract inhibited the increase of cytosolic free calcium induced by high doses of glutamate. Similar results were observed in experiments with primarily cultured hippocampal neurons of neonatal rats. The authors concluded that the water extract of Poria cocos exerts bidirectional regulation of cytosolic free calcium in brain nerve cells.

The antagonistic effects of Poria cocos on the ototoxicity of kanamycin in guinea-pigs was studied by Liu et al. [71], who infused the decoction into the guinea-pigs and compared the differences in the general intoxicating symptom, the prayer's reflex threshold, brainstem auditory evoked potentials, and the absence rate of outer hair cells in the first turn of the cochlea. The authors con- cluded that Poria cocos may serve as a good antagonistic agent in kanamycin treatment.

Tai et al. [13] studied the anti-emetic properties of an ethanolic extract from Poria cocos and 17 triterpenoids (11 natural products isolated from the fungus and 6 semisynthetic derivatives). Using copper sulfate as an emetic agent in frogs, the authors demonstrated that the ethanolic extract, along with $\mathbf{1}$, its methyl ester, 7, and its dimethyl derivative all had anti-emetic properties; however, the last compound exhibited the highest activity at the lowest dose. In addition, $\mathbf{1 3}$ showed a high degree of toxicity. The authors concluded that triterpenes with an exo-methylene group at $\mathrm{C}_{24}$ in their side-chain (eburicanes) have anti-emetic properties.

Lee et al. [72] recently studied the effects of $\mathbf{1}$, dehydroeburicoic acid (17) (the names of these compounds are changed here with respect to the formula), and 4 (called $3 \beta$-hydroxylanosta-7,9 (11),24-trien-21-oic acid in this paper) on human 5-hydroxytryptamine $3 \mathrm{~A}\left(5-\mathrm{HT}_{3 \mathrm{~A}}\right)$ receptor channel activity. The three triterpenoids inhibited the 5HT-induced inward current $\left(I_{5-\mathrm{HT}}\right)$ in a concentration dependent and reversible manner, with $\mathrm{IC}_{50}$ values of $3.2,5.5$, and $1.4 \mu \mathrm{M}$, respectively. While the inhibition of $I_{5-\mathrm{HT}}$ by the triterpenoids occurred in a noncompetitive manner, the inhibition by $\mathbf{1}$ and $\mathbf{4}$ showed greater voltage-dependency. These results indicate that triterpenoids may regulate the expressed 5- $\mathrm{HT}_{3 \mathrm{~A}}$ receptors in Xenopus oocytes and that this regulation may be partly responsible for the pharmacological effects of Poria cocos. These results suggest that these compounds could be used in therapeutics for the suppression of clinical symptoms related with activity of $5-\mathrm{HT}_{3 \mathrm{~A}}$ receptor, such as vomiting and visceral pain.

Wang et al. [73] isolated a water-insoluble $\beta$-( $1 \rightarrow 3)$-D-glucan from the sclerotium of Poria cocos and synthesized a carboxymethylated derivative. They demonstrated that carboxymethylation enhances the bile acid binding capacity of the polysaccharides in vitro, probably due to the improved water solubility and structural changes caused by carboxymethylation. In addition, the semisynthetic derivative exhibited an increased antioxidant activity with respect to the original compound. The authors hypothesized that the carboxymethylated derivative could provide several health benefits, including reduction of cholesterol and blood pressure.

The antioxidant effects of a water soluble extract from Poria cocos (PCW) and its effect on $\beta$-amyloid-induced neurotoxicity on rat pheochromocytoma (PC12) cells was studied by Park et al. [74], who suggested that PCW may protect cells by suppressing both the oxidative stress and the apoptosis induced by $\beta$-amyloid (142). Indeed, the pretreatment of PC12 cells with PCW within the concentration range of $5-125 \mu \mathrm{g} / \mathrm{mL}$ reduced $\beta$-amyloid (1-42)induced cell death. In addition, cells treated with PCW attenuated $\beta$-amyloid (1-42)-induced cytotoxicity, apoptotic features, and accumulation of intracellular oxidative damage while decreasing the expression of the apoptotic protein Bax and the activity of caspase-3. It also enhanced the expression of anti-apoptotic protein $\mathrm{Bcl}-2$. Taken together, these findings suggest that PCW may be a potential natural agent against Alzheimer's disease.

\section{Pharmacokinetic Studies and Therapeutic Use} $\nabla$

Poria cocos is a well-known traditional Chinese medicine used for its diuretic, sedative, and tonic effects [32]; indeed, it has been demonstrated to have spleen-invigorative, stomach-tonifying, 
sedative, tranquilizing, diuretic, and damp-clearing effects. It is mainly used to treat retention of phlegm and fluid, dysuria, edema, poor appetite with watery stool, palpitations, and insomnia [7]. There are different specifications in the traditional use depending on the crude drug used; for example, fu-ling-pi promotes diuresis and reduces swelling whereas chih-fu-ling cleanses heat and eliminates dampness [6].

Poria cocos has no adverse reactions described in the literature; however, it is not recommended in the case of polyuria.

Using the human colonic adenocarcinoma (Caco-2) cell monolayer as an intestinal epithelial cell model, Zheng and Yang [75] studied the permeability of $\mathbf{1 3}, \mathbf{1 6}$, and $6 \alpha$-hydroxypolyporenic acid C (18) from the apical to the basolateral side and vice-versa. The transport parameters and apparent permeability coefficient values of $\mathbf{1 3}$ and $\mathbf{1 6}$ were nearly of the same magnitude as those of the standard drug propranolol while those for $\mathbf{1 8}$ lay between those of propranolol and atenolol (the reference drugs used in the experiments). Moreover, the efflux transport of $\mathbf{1 3}$ and $\mathbf{1 6}$ were higher than their influx transport. The authors concluded that while all three compounds can be absorbed across intestinal epithelial cells, $\mathbf{1 3}$ and $\mathbf{1 6}$ could be completely absorbed whereas 18 could only be moderately absorbed.

In a similar study, the same authors [76] demonstrated that 1 could be transported through the Caco-2 cell monolayer in a concentration-dependent manner and that the transport was linear with time. The absorption in the apical to basolateral direction and the secretion in the opposite direction were both poor, with the transport parameters and apparent permeability coefficient values being comparable to those of the reference drug atenolol. Although the diffusion of 1 was passive, ATP was partially involved in its transport.

\section{Commercial Products and Form of Administration $\nabla$}

Poria cocos is present in the market under the names fu-ling, hoelen, poria, tuckahoe, and Indian bread. It is normally white in color ("white poria"), but there is a light red variant called "red poria". In China, Poria cocos is one of the major ingredients of $\mathrm{Bu}$ Chy, a drug which has long been used to aid in the recovery from chronic diseases. The sclerotium is presented in different commercial forms such as dry power and capsules. It is available in Western markets as a phytomedicine or as a natural dietary supplement, and is either presented alone or in combination with other medicinal plants or fungi. Dry powder of Poria cocos sclerotium is used in infusions at daily doses of $6-18 \mathrm{~g}$. For reinforcing the spleen and the stomach, the recommended daily dose is 9 to $18 \mathrm{~g}$ whereas for edema the dose may range as high as 30 to $45 \mathrm{~g}$. As a sedative or for the treatment of palpitations and insomnia, the recommended dose is from 3 to $9 \mathrm{~g}[6,7]$.

\section{Conclusions}

\section{$\nabla$}

Several pharmacological effects of Poria cocos have been clearly demonstrated in experimental studies. Of these, it is important to distinguish between anti-inflammatory and immunostimulant effects. Although the crude drug has been studied with regard to both properties, experimental studies show that purified extracts achieve the best effect. Thus, while anti-inflammatory activity has been clearly demonstrated for the triterpene-en- riched fraction, immunomodulation is correlated with the polysaccharide-enriched fractions. In the case of anticancer properties, both groups of compounds are implicated, triterpenes as cytotoxic principles and polysaccharides as immunostimulants. With respect to clinical data, no relevant reports on the extract or its isolated compounds are generally cited, only studies carried out within traditional Chinese medicine; however, these usually employ drug mixtures, such as STA-1 (a mixture of 10 crude drugs) for treating allergic asthma [77] or Yi-jin (a mixture with 3 other crude drugs) for treating diabetes mellitus type II [78]. With regard to the potential cytotoxicity of Poria cocos, no reports have appeared in the literature. This fungus is usually administered in China at high doses (up to $45 \mathrm{~g}$ ), with no specification of adverse effects, although the Chinese Pharmacopoeia lists several contraindications, such as in cases of polyuria, spermatorrhoea, or urogenital prolapse [79].

\section{References}

1 Lindner DL, Banik MT. Molecular phylogeny of Laetiporus and other brown rot polypore genera in North America. Mycologia 2008; 100 : 417-430

2 Binder M, Hibbett DS, Larsson KH, Larsson E, Langer E, Langer G. The phylogenetic distribution of resupinate forms across the major clades of mushroom-forming fungi (Homobasidiomycetes). Syst Biodivers 2005; 3: 113-157

3 Ryvarden L. Genera of polypores: nomenclature and taxonomy. Synopsis fungorum 5. Oslo: Fungiflora; 1991: 363

4 Giner-Larza EM, Máñez S, Giner-Pons RM, Carmen Recio M, Ríos JL. On the anti-inflammatory and anti-phospholipase $A_{2}$ activity of extracts from lanostane-rich species. J Ethnopharmacol 2000; 73: 61-69

5 Illana-Esteban C. Interés medicinal de Poria cocos (= Wolfiporia extensa). Rev Iberoam Micol 2009; 26: 103-107

6 Hsu HY, Chen YP, Shen SJ, Hsu CS, Chen CC, Chang HC. Oriental Materia Medica. A concise guide. Long Beach: Oriental Healing Arts Institute; 1986: 305-306

7 Chang HM, But PPH. Pharmacology and applications of Chinese Materia Medica. Singapore: World Scientific; 1987: 875-877

8 Tai T, Akahori A, Shingu T. Triterpenoids from Poria cocos. Phytochemistry 1991; 30: 2796-2797

9 Tai T, Akahori A, Shingu T. A lanostane triterpenoid from Poria cocos. Phytochemistry 1992; 31: 2548-2549

10 Tai T, Akahori A, Shingu T. Triterpenes of Poria cocos. Phytochemistry 1993; 32: 1239-1244

11 Tai T, Shingu T, Kikuchi T, Tezuka Y, Akahori A. Triterpenes from the surface layer of Poria cocos. Phytochemistry 1995; 39: 1165-1169

12 Tai T, Shingu T, Kikuchi T, Tezuka Y, Akahori A. Isolation of lanostanetype triterpene acids having an acetoxyl group from sclerotia of Poria cocos. Phytochemistry 1995; 40: 225-231

13 Tai T, Akita Y, Konoshita K, Koyama K, Takahashi K, Watanabe K. Antiemetic principles of Poria cocos. Planta Med 1995; 61: 527-530

14 Akihisa T, Nakamura Y, Tokuda H, Uchiyama E, Suzuki T, Kimura Y, Uchikura K, Nishi $H$. Triterpene acids from Poria cocos and their anti-tumorpromoting effects. J Nat Prod 2007; 70: 948-953

15 Akihisa T, Uchiyama E, Kikuchi T, Tokuda H, Suzuki T, Kimura Y. Anti-tumor-promoting effects of 25-methoxyporicoic acid A and other triterpene acids from Poria cocos. J Nat Prod 2009; 72: 1786-1792

16 Zheng Y, Yang XW. Two new lanostane triterpenoids from Poria cocos. J Asian Nat Prod Res 2008; 10: 323-328

17 Zheng Y, Yang XW. Poriacosones A and B: two new lanostane triterpenoids from Poria cocos. J Asian Nat Prod Res 2008; 10: 645-651

18 Yasukawa K, Kaminaga T, Kitanaka S, Tai T, Nunoura Y, Natori S, Takido M. 3ß-p-hydroxybenzoyldehydrotumulosic acid from Poria cocos, and its anti-inflammatory effects. Phytochemistry 1998; 48: 1357-1360

19 Nukaya H, Yamashiro H, Fuzakawa H, Ishida H, Tsuji K. Isolation of inhibitors of TPA-induced mouse ear edema from hoelen, Poria cocos. Chem Pharm Bull 1996; 44: 847-849

20 Akihisa T, Mizushina Y, Ukiya M, Oshikubo M, Kondo S, Kimura Y, Suzuki T, Tai T. Dehydrotrametenonic acid and dehydroeburiconic acid from Poria cocos and their inhibitory effects on eukaryotic DNA polymerase $\alpha$ and $\beta$. Biosci Biotechnol Biochem 2004; 68: 448-450 
21 Yokoyama A, Natori S, Aoshima K. Distribution of tetracyclic triterpenoids of lanostane group and sterols in the higher fungi especially of the Polyporaceae and related families. Phytochemistry 1975; 14: 487497

22 Zheng $Y$, Yang XW. Absorption of triterpenoid compounds from Indian bread (Poria cocos) across human intestinal epithelial (Caco-2) cells in vitro. Zhongguo Zhong Yao Za Zhi 2008; 33: 1596-1601

23 Ukiya M, Akihisa T, Tokuda H, Hirano M, Oshikubo M, Nobukuni Y, Kimura Y, Tai T, Kondo S, Nishino $\mathrm{H}$. Inhibition of tumor-promoting effects by poricoic acids $\mathrm{G}$ and $\mathrm{H}$ and other lanostane-type triterpenes and cytotoxic activity of poricoic acids A and G from Poria cocos. J Nat Prod 2002; 65: 462-465

24 Chihara G, Hamuro J, Maeda Y, Arai Y, Fukuoka F. Antitumor polysaccharide derived chemically from natural glucan (pachyman). Nature 1970; 225: 943-944

25 Kanayama $\mathrm{H}$, Adechi N, Togami M. A new antitumor polysaccharide from the mycelia of Poria cocos Wolf. Chem Pharm Bull 1983; 31: $1115-1118$

26 Jin Y, Zhang L, Chen L, Chen Y, Cheung PC, Chen L. Effect of culture media on the chemical and physical characteristics of polysaccharides isolated from Poria cocos mycelia. Carbohydr Res 2003; 338: 1507-1515

27 Wang Y, Zhang M, Ruan D, Shashkov AS, Kilcoyne M, Savage AV, Zhang L. Chemical components and molecular mass of six polysaccharides isolated from the sclerotium of Poria cocos. Carbohydr Res 2004; 339: 327-334

28 Huang $Q$ Zhang $L$. Solution properties of $(1 \rightarrow 3)-\alpha$-D-glucan and its sulfated derivative from Poria cocos mycelia via fermentation tank. Biopolymers 2005; 79: 28-38

29 Li G, Xu ML, Lee CS, Woo MH, Chang HW, Son JK. Cytotoxicity and DNA topoisomerases inhibitory activity of constituents from the sclerotium of Poria cocos. Arch Pharm Res 2004; 27: 829-833

30 Cuéllar MJ, Giner RM, Recio MC, Just MJ, Máñez S, Ríos JL. Effect of the basidiomycete Poria cocos on experimental dermatitis and other inflammatory conditions. Chem Pharm Bull (Tokyo) 1997; 45: 492-494

31 Giner EM, Máñez S, Recio MC, Giner RM, Cerdá-Nicolás M, Ríos JL. In vivo studies on the anti-inflammatory activity of pachymic and dehydrotumulosic acids. Planta Med 2000; 66: 221-227

32 Cuéllar MJ, Giner RM, Recio MC, Just MJ, Máñez S, Ríos JL. Two fungal lanostane derivatives as phospholipase A2 inhibitors. J Nat Prod 1996; 59 : 977-979

33 Jain MK, Yu B, Rogers JM, Smith AE, Boger ETA, Ostrander RL, Rheingold $A L$. Specific competitive inhibitor of secreted phospholipase $A_{2}$ from berries of Schinus terebinthifolius. Phytochemistry 1995; 39: 537-547

34 Scott DL, White SP, Otwinowski Z, Yuan W, Gelb MH, Sigler PB. Interfacial catalysis: the mechanism of phospholipase $A_{2}$. Science 1990; 250 : 1541-1546

35 Kaminaga T, Yasukawa K, Takido M, Tai T, Nunoura Y. Inhibitory effects of Poria cocos on 12-O-tetradecanoylphorbol-13-acetate-induced eat oedema and tumour promotion in mouse skin. Phytother Res 1996; 10: $581-584$

36 Prieto JM, Recio MC, Giner RM, Máñez S, Giner-Larza EM, Ríos JL. Influence of traditional Chinese anti-inflammatory medicinal plants on leukocyte and platelet functions. J Pharm Pharmacol 2003; 55: 12751282

37 Fuchs SM, Heinemann C, Schliemann-Willers S, Härtl H, Fluhr JW, Elsner $P$. Assessment of anti-inflammatory activity of Poria cocos in sodium lauryl sulphate-induced irritant contact dermatitis. Skin Res Technol 2006; 12: 223-227

38 Spelman K, Burns JJ, Nichols D, Winters N, Ottersberg S, Tenborg M. Modulation of cytokine expression by traditional medicines: a review of herbal immunomodulators. Altern Med Rev 2006; 11: 128-150

$39 \mathrm{Yu}$ SJ, Tseng J. Fu-Ling, a Chinese herbal drug, modulates cytokine secretion by human peripheral blood monocytes. Int J Immunopharmacol 1996; 18: $37-44$

40 Tseng J, Chang JG. Suppression of tumor necrosis factor- $\alpha$, interleukin$1 \beta$, interleukin- 6 and granulocyte-monocyte colony stimulating factor secretion from human monocytes by an extract of Poria cocos. Chin J Microbiol Immunol 1992; 1: 1-10

41 Chang $\mathrm{HH}$, Yeh $\mathrm{CH}$, Sheu F. A novel immunomodulatory protein from Poria cocos induces Toll-like receptor 4-dependent activation within mouse peritoneal macrophages. J Agric Food Chem 2009; 57: 61296139
42 Chen $X$, Zhang $L$, Cheung PC. Immunopotentiation and anti-tumor activity of carboxymethylated-sulfated $\beta$ - $(1 \rightarrow 3)$-D-glucan from Poria cocos. Int Immunopharmacol 2010; 10: 398-405

43 Kaminaga T, Yasukawa K, Kanno T, Nunoura Y, Takido M. Inhibitory effects of lanostane-type triterpene acids, the components of Poria cocos, on tumor promotion by 12-0-tetradecanoylphorbol-13-acetate in two-stage carcinogenesis in mouse skin. Oncology 1996; 53: 382-385

44 Mizushina Y, Akihisa T, Ukiya M, Murakami C, Kuriyama I, Xu X, Yoshida $H$, Sakaguchi K. A novel DNA topoisomerase inhibitor: dehydroebriconic acid; one of the lanostane-type triterpene acids from Poria cocos. Cancer Sci 2004; 95: 354-360

45 Kang HM, Lee SK, Shin DS, Lee MY, Han DC, Baek NI, Son KH, Kwon BM. Dehydrotrametenolic acid selectively inhibits the growth of $\mathrm{H}$-ras transformed rat2 cells and induces apoptosis through caspase- 3 pathway. Life Sci 2006; 78: 607-613

46 Gapter L, Wang Z, Glinski J, Ng KY. Induction of apoptosis in prostate cancer cells by pachymic acid from Poria cocos. Biochem Biophys Res Commun 2005; 332: 1153-1161

47 Ling H, Zhou L, Jia X, Gapter LA, Agarwal R, Ng KY. Polyporenic acid C induces caspase-8-mediated apoptosis in human lung cancer A549 cells. Mol Carcinogen 2009; 48: 498-507

$48 \mathrm{Wu} \mathrm{SJ}, \mathrm{Ng} \mathrm{LT}$, Lin CC. Antioxidant activities of some common ingredients of traditional Chinese medicine, Angelica sinensis, Lycium barbarum and Poria cocos. Phytother Res 2004; 18: 1008-1012

49 Schinella GR, Tournier HA, Prieto JM, Mordujovich de Buschiazzo P, Ríos $J L$. Antioxidant activity of anti-inflammatory plant extracts. Life Sci 2002; 70: 1023-1033

50 Zhou L, Zhang Y, Gapter LA, Ling H, Agarwal R, Ng KY. Cytotoxic and antioxidant activities of lanostane-type triterpenes isolated from Poria cocos. Chem Pharm Bull (Tokyo) 2008; 56: 1459-1462

51 Yance DR, Sagar SM. Targeting angiogenesis with integrative cancer therapies. Integr Cancer Ther 2006; 5: 9-29

52 Lee $K Y$, Jeon YJ. Polysaccharide isolated from Poria cocos sclerotium induces NF-KB/Rel activation and iNOS expression in murine macrophages. Int Immunopharmacol 2003; 3: 1353-1362

53 Lee KY, You HJ, Jeong HG, Kang JS, Kim HM, Rhee SD, Jeon YJ. Polysaccharide isolated from Poria cocos sclerotium induces NF-KB/Rel activation and iNOS expression through the activation of p 38 kinase in murine macrophages. Int Immunopharmacol 2004; 4: 1029-1038

54 Jin Y, Zhang L, Zhang M, Chen L, Cheung PC, Oi VE, Lin Y. Antitumor activities of heteropolysaccharides of Poria cocos mycelia from different strains and culture media. Carbohydr Res 2003; 338: 1517-1521

55 Zhang M, Chiu LC, Cheung PC, Oi VE. Growth-inhibitory effects of a $\beta$ glucan from the mycelium of Poria cocos on human breast carcinoma MCF-7 cells: cell-cycle arrest and apoptosis induction. Oncol Rep 2006; 15: 637-643

56 Wang Y, Zhang L, Li Y, Hou X, Zeng F. Correlation of structure to antitumor activities of five derivatives of a $\beta$-glucan from Poria cocos sclerotium. Carbohydr Res 2004; 339: 2567-2574

57 Zhang L, Chen L, Xu X, Zeng F, Cheung PC. Effect of molecular mass on antitumor activity of heteropolysaccharide from Poria cocos. Biosci Biotechnol Biochem 2005; 69: 631-634

58 Lin Y, Zhang L, Chen L, Jin Y, Zeng F, Jin J, Wan B, Cheung PC. Molecular mass and antitumor activities of sulfated derivatives of $\alpha$-glucan from Poria cocos mycelia. Int J Biol Macromol 2004; 34: 289-294

59 Chen YY, Chang HM. Antiproliferative and differentiating effects of polysaccharide fraction from fu-ling (Poria cocos) on human leukemic U937 and HL-60 cells. Food Chem Toxicol 2004; 42: 759-769

$60 \mathrm{Li} \mathrm{TH}$, Hou CC, Chang CLT, Yang WC. Anti-hyperglycemic properties of crude extract and triterpenes from Poria cocos. Evid Based Complement Alternat Med, advance online publication 16 September 2010; doi: $10.1155 / 2011 / 128402$

61 Sato M, Tai T, Nunoura Y, Yajima Y, Kawashima S, Tanaka K. Dehydrotrametenolic acid induces preadipocyte differentiation and sensitizes animal models of noninsulin-dependent diabetes mellitus to insulin. Biol Pharm Bull 2002; 25: 81-86

$62 \mathrm{Su} Y B$, Huang YT. Poria cocos inhibited the activation of hepatic stellate cells. Planta Med 2009; 75: 1034-1035

63 Huang YC, Chang WL, Huang SF, Lin CY, Lin HC, Chang TC. Pachymic acid stimulates glucose uptake through enhanced GLUT4 expression and translocation. Eur J Pharmacol 2010; 648: 39-49 
64 Hattori T, Hayashi K, Nagao T, Furuta K, Ito M, Suzuki Y. Studies on antinephritic effects of plant components (3): effect of pachyman, a main component of Poria cocos Wolf on original-type anti-GBM nephritis in rats and its mechanisms. Jpn J Pharmacol 1992; 59: 89-96

65 Dhan HP, Hou AJ, Lu FE, Huang JL. Experimental study on the inhibitory effect of carboxymethyl pachymaram on hepatitis B virus expression from transfected cells. Zhonghua Shi Yan He Lin Chuang Bing Du Xue Za Zhi 2005; 19: 290-292

66 Schinella GR, Tournier HA, Prieto JM, Ríos JL, Buschiazzo H, Zaidenberg A. Inhibition of Trypanosoma cruzi growth by medical plant extracts. Fitoterapia 2002; 73: 569-575

67 Li GH, Shen YM, Zhang KQ. Nematicidal activity and chemical component of Poria cocos. J Microbiol 2005; 43: 17-20

68 Zhang GW, Liu HY, Xia QM, Li JQ Lü H, Zhang QH, Yao ZF. Anti-rejection effect of ethanol extract of Poria cocos Wolf in rats after cardiac allograft implantation. Chin Med J (Engl) 2004; 117: 932-935

69 Sekiya N, Goto H, Shimada Y, Endo Y, Sakakibara I, Terasawa K. Inhibitory effects of triterpenes isolated from Hoelen on free radical-induced lysis of red blood cells. Phytother Res 2003; 17: 160-162

70 Chen W, An W, Chu J. Effect of water extract of Poria on cytosolic free calcium concentration in brain nerve cells of neonatal rats. Zhongguo Zhong Xi Yi Jie He Za Zhi 1998; 18: 293-295

71 Liu YC, Liu GY, Liu RL. Effects of Poria cocos on ototoxicity induced by kanamycin in guinea-pigs. Zhongguo Zhong Xi Yi Jie He Za Zhi 1995; 15: $422-423$
72 Lee JH, Lee YJ, Shin JK, Nam JW, Nah SY, Kim SH, Jeong JH, Kim Y, Shin M, Hong M, Seo EK, Bae H. Effects of triterpenoids from Poria cocos Wolf on the serotonin type $3 \mathrm{~A}$ receptor-mediated ion current in Xenopus oocytes. Eur J Pharmacol 2009; 615: 27-32

73 Wang Y, Yu Y, Mao J. Carboxymethylated $\beta$-glucan derived from Poria cocos with biological activities. J Agric Food Chem 2009; 57: 1091310915

74 Park YH, Son IH, Kim B, Lyu YS, Moon HI, Kang HW. Poria cocos water extract (PCW) protects PC12 neuronal cells from beta-amyloid-induced cell death through antioxidant and antiapoptotic functions. Pharmazie 2009; 64: 760-764

75 Zheng $Y$, Yang XW. Absorption of triterpenoid compounds from Indian bread (Poria cocos) across human intestinal epithelial (Caco-2) cells in vitro. Zhongguo Zhong Yao Za Zhi 2008; 33: 1596-1601

76 Zheng Y, Yang XW. Absorption and transport of pachymic acid in the human intestinal cell line Caco-2 monolayers. Zhong Xi Yi Jie He Xue Bao 2008; 6: 704-710

77 Chang TT, Huang CC, Hsu CH. Clinical evaluation of the Chinese herbal medicine formula STA-1 in the treatment of allergic asthma. Phytother Res 2006; 20: 342-347

78 Jia $W$, Gao W, Tang L. Antidiabetic herbal drugs officially approved in China. Phytother Res 2003; 17: 1127-1134

79 Manual Práctico de Farmacopea y Prescripción en Medicina Tradicional China. Amposta: AISVI; 1993: 67 